\title{
Inheritance of Isoenzymes in European Beech (Fagus sylvatica L.)
}

\author{
G. Müller-Starck and R. Starke
}

Segregation of isoenzymes was studied among 34 full-sib families of Fagus sy/vatica L. by means of gel electrophoresis. Of the 16 enzyme systems analyzed, two showed substantial tissue-specific expression of isoenzymes. The remaining 14 enzyme systems are controlled genetically by at least 20 polymorphic gene loci, three of which were inferred from additional population studies. The inheritance of the complex system of 6PGDH is studied in detail. A 20-locus nomenclature is suggested, including 78 codominant alleles. Analyses of two-locus combinations did not reveal linkage between any of the tested gene loci.

European beech (Fagus sylvatica L.) covers a large continuous geographic range in Europe. It is a carrier tree species of various forest ecosystems and fulfills a wide spectrum of environmental, social, and economic functions. In spite of the long tradition in silvicultural research and in the study of beech provenances, genetic characterization of beech populations started not much longer ago than one decade (Kim 1980). Since then, approximately 300 beech populations have been studied by employing isoenzyme markers (for review see Müller-Starck et al. 1992) exclusively. Moreover, compared to many other deciduous trees or conifers, a surprisingly low number of gene loci has been used. There are 14 studies on genetic variation in beech populations, which comprise a total of nearly 15,000 individuals, but the majority of these individuals were genotyped on the basis of three polymorphic loci. Two studies refer to six loci. Recent studies by Müller-Starck include more loci because they refer to the results of the investigation reported here.

There is little information available on

From the Abteilung Forstgenetik und Forstpflanzenzüchtung, Universität Göttingen, Göttingen, Germany (Müller-Starck and Starke), and the Eidgenöss. Forschungsanstalt für Wald, Schnee und Landschaft, $\mathrm{CH}$ 8903 Birmensdorf-ZH, Switzerland (Müller-Starck). The technical assistance of M. Günther and $\mathrm{G}$. Dinkel is greatly appreciated. We wish to thank F. Bergmann, H. H. Hattemer, H. R. Gregorius, M. Ziehe, and two unknown referees for very useful comments. The help of $E$. Gillet in the calculation of the exact levels of significance is greatly appreciated. This study was supported financially by the Deutsche Forschungsgemeinschaft, Bad Godesberg, and by the Bundesministerium für Forschung und Technologie, Bonn.

Journal of Heredity 1993;84:291-296; 0022-1503/93/\$5.00 five gene loci, two (Got-A, and $L a p-A$ ) reveal tissue-unspecific expression.

Merzeau et al. (1989) analyzed segregation among offspring of heterozygous trees and inferred genetic control and inheritance with respect to the loci $M d h-1$, 6Pgd-1, Pgi-1 (=Mdh-B, 6Pgdh-A, Pgi-B), and Sod-1 (SOD = superoxide dismutase). Evidence for other loci ( $A c P-1, I d h-1, P x-3$, $S k d h-1$ ) could not explicitly be tested.

The objective is to proceed in verifying the inheritance of isoenzymes in beech and thus to contribute to a better use of gene markers in forest genetic studies.

\section{Materials and Methods}

\section{Controlled Crosses}

During 1980 and 1986, we performed crosses among intensively flowering trees in parks of Göttingen or its vicinity. For each crossing, a minimum of 80 cross-fertilized and 120 self-fertilized female flowers were isolated using water-resistant but air-permeable bags. Pollen donor trees were chosen on the basis of their isoenzyme phenotypes (carriers of rare variants were preferred). We collected branches with male flowers a few days before pollen shedding. In order to avoid contamination by external pollen, these branches were rinsed in the greenhouse and kept separate from each other. For ripening of pollen, temperature was $25^{\circ} \mathrm{C}$ and humidity was greater than $50 \%$ to prevent the breakdown of the ripening process of pollen. Pollination was performed twice with in- 
Table 1. Survey of 34 full-sib familles with at least 20 analyzed embryos per cross $(N)^{a}$

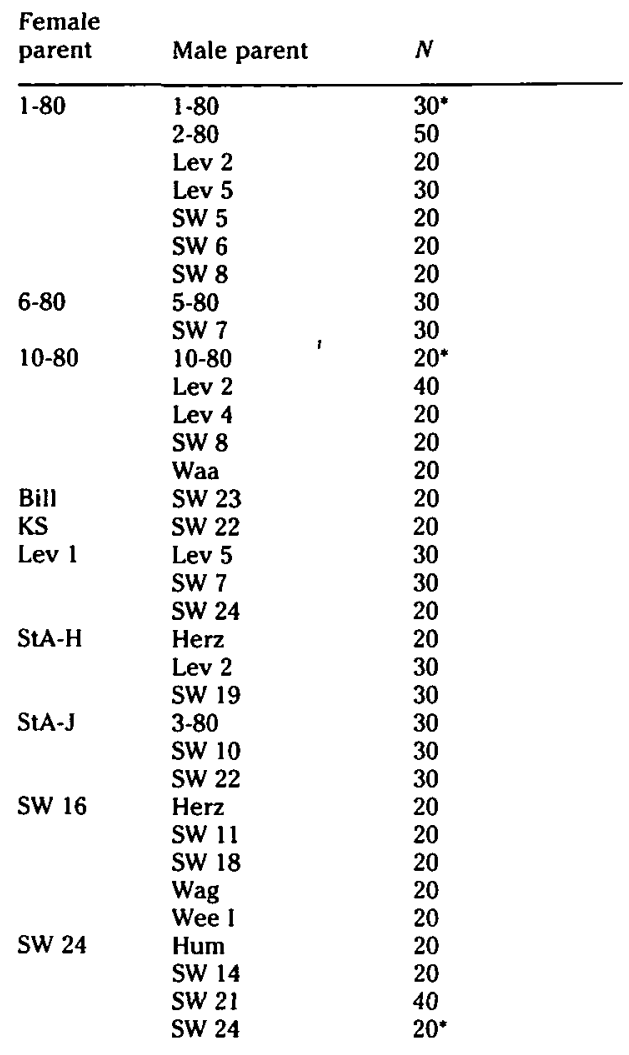

asterisks indicate offspring from self-fertilization.

clusion of self-fertilization and reciprocal crossings.

We collected beech nuts at the end of September and stored them for 4-6 weeks at a temperature of $3^{\circ} \mathrm{C}-5^{\circ} \mathrm{C}$ to overcome dormancy dependent inhibition of enzyme activity. Preliminary studies on the germination capability indicated low germination percentages $(40 \%-80 \%)$. To avoid bias in the genetic analyses due to viability selection following germination, we genotyped seeds (embryos) instead of seedlings.

Table 1 shows the full-sib families surveyed with a minimum of 20 analyzed individuals. Sample sizes are small because female flowers tend to be scattered in low density over the upper and outside parts of beech crowns so that crosses are extremely laborious. Besides that, extraction of pollen from anthers and determination of duration of receptivity of female flowers are difficult and are not generally performed (Nielsen and Schaffalitzky de Muckadell 1954). Finally, losses caused by seed parasites (Laspeyresia grossana Haworth) cannot be avoided.

Table 2. Survey of electrophoretic methods used for verification of isoenzymes in embryos, buds, young leaves, and pollen

\begin{tabular}{llllll} 
Enzyme system $^{\circ}$ & Buffer $^{b}$ & Enzyme system & Buffer & Enzyme system & Buffer \\
\hline ACP & 1,2 & IDH & 4,5 & PEPCA & 4,5 \\
ACO & 3 & LAP & 1 & 6 PGDH & 3,4 \\
DIA & 4,3 & MDH & 4,5 & PGI & 1 \\
EST & 1,2 & NDH & 4,5 & PGM & 4 \\
GDH & 3 & PER & 2 & SKDH & 4,5 \\
GOT & 2 & & & &
\end{tabular}

No. Electrode buffer/pH

Gel buffer/pH

$1 \quad 0.05 \mathrm{M} \mathrm{LiOH}-0.19 \mathrm{M}$ boric acid/8.1

$0.05 \mathrm{M}$ Tris- $0.01 \mathrm{M}$ citric acid/8.1 (with $10 \%$ electrode buffer) (Source: Lundkvist, 1979)

$2 \quad 0.06 \mathrm{M} \mathrm{NaOH}-0.30 \mathrm{M}$ boric acid/8.0

0.07 M Tris-0.02 M HCl/8.7 (Source: Poulik, 1957 , modified)

$3 \quad 0.14 \mathrm{M}$ Tris- $0.04 \mathrm{M}$ citric acid/7.8

Diluted electrode buffer (2.5:1)

$0.14 \mathrm{M}$ Tris $-0.04 \mathrm{M}$ citric acid $/ 7.0$

Diluted electrode buffer (2.5:1)

$5 \quad 0.14 \mathrm{M}$ Tris- $0.04 \mathrm{M}$ citric acid/7.0

$0.04 \mathrm{M}$ Tris $0.001 \mathrm{M}$ EDTA and $0.05 \mathrm{M}$ histidine $\mathrm{HCl} / 5.7$

- For designation of enzyme systems and EC no., see Table 4.

- Electrode and gel buffers used for starch gel electrophoresis:

$c=$ Aspartate aminotransferase (AAT).

a Bergmann and Scholz (1989)

e Embryos.

\section{Electrophoretic Methods}

We separated enzymes from crude homogenate of embryos, buds, young leaves, or pollen by standard horizontal starch gel electrophoresis. For the extraction method, see Müller-Starck (1985, 1989). For composition of buffers and enzyme systems see Table 2. Starch gel-concentration is $11 \%$ (microwave-supported preparation), voltage distribution is $20-30 \mathrm{~V} / \mathrm{cm}$, and bridge distance is $12 \mathrm{~cm}$. For DIA and PER the high-resolution power of isoelectric focusing (IEF) is favorable: $6 \%$ poly acrylamide gels with mixed carrier ampholytes (80\% pH $3.5-10,20 \%$ pH $3.5-5)$. Desalting of the crude extracts is sug gested. Buffers are $0.5 \mathrm{M} \mathrm{H}_{3} \mathrm{PO}_{4}$ for the anode and $0.5 \mathrm{M} \mathrm{NaOH}$ for the cathode. The run is started at $160 \mathrm{~V}(0.5 \mathrm{~h}$ prefocusing) and stopped after $2.5 \mathrm{~h}$ at approximately $2500 \mathrm{~V}$.

For buffer formulations for enzyme stains, see Müller-Starck (1985). By omitting enzyme substrates parallel to the regular stains, we checked for each enzyme system whether the isoenzyme variants in the zymogram in fact referred to the enzyme system under consideration or if substrate unspecificity occurred. Tissuespecific expression of isoenzymes was studied with respect to pollen, bud, and leaf samples of 33 parental trees (see Table 1).

\section{Segregation Studies}

We verified the mode of inheritance of isoenzymes by testing the hypothesis of con- formity of the segregation observed among the embryos from controlled crosses with the expected Mendelian segregations. Isoenzyme variants that followed Mendelian segregations were considered to represent an allele at one of the enzyme-coding gene loci and thus to be applicable as a species-specific gene marker. Recombination was tested by utilizing full-sibs with at least one double heterozygote parent. Four hypotheses were tested: regular segregation of (1) both locus types; (2) the single-locus types at the first gene locus; (3) the single-locus types at the second gene locus; and (4) the summed "recombinant" and the summed "nonrecombjnant" genotypes.

For each cross, we compared statistically the observed and expected genotypic segregations by determining the exact level of significance (modeled after R. A. Fischer's Exact Test of a $2 \times 2$ contingency table): each possible distribution of genotypes of the same size is generated, and its probability under the given hypothesis is calculated. All probabilities less than or equal to the probability of the observed genotypic distribution are added up to give the exact level of significance $P$. The hypothesis is rejected if $P$ is less than or equal to a certain set value. In accordance with common usage, $P>.05$ is considered nonsignificant, while smaller values of $P$ are significant with the usual number of asterisks $\left(^{*}=P \leq .05,{ }^{* *}=P \leq .01,{ }^{* * *}=\right.$ $P \leq .001)$. As its name implies, this test calculates the exact level of significance 
that is only approximated - for small sample sizes rather poorly-by asymptotic tests of goodness-of-fit, such as $\chi^{2}$ - and log-likelihood-ratio $(G)$ tests.

Two-locus genotypes, which could not be distinguished from each other, were pooled. The number of different identifiable two-locus genotypes was relevant for the calculation of the exact significance levels. If both parents were double heterozygous, statistical tests were calculated for each parent separately.

\section{Survey of Isoenzyme Polymorphism}

Because the available full-sibs represent only a portion of the isoenzyme polymorphism in beech populations, two classes of isoenzymes were surveyed: (1) isoenzymes represented in full-sib families (see arrows in Figure 1) and (2) isoenzymes monitored in 19 beech populations, which includes 2,381 individuals (samples of airpolluted juvenile and adult populations; see Müller-Starck 1989; Müller-Starck and Ziehe 1991). Genetic types are designated by letters for gene loci and by numbers for alleles. As in Figure 1, the letter $A$ in Table 3 and Table 6 refers to that locus, the alleles of which are apparent within the zone of the zymogram with the largest relative mobility. Succeeding letters designate the following zones in decreasing order of relative mobility. The letter $A$ is also used for enzyme systems that are apparently represented in the zymogram by only one zone.

\section{Results and Discussion}

\section{Tissue-specific Expression of Isoenzymes}

Acid phosphatases (ACP) and esterases (EST) can show variation in the isoenzyme patterns between buds, leaves, and pollen of one and the same individual to such an extent that genotyping is questionable. The same holds for embryos and succeeding seedling stages. Therefore, both systems are not generally subjects of inheritance studies. In the case of peroxidases (PER), only the fast migrating zone (Per-A) belongs to this category, while the slow migrating zone (Per-B, see Figure 1) is expressed tissue unspecific in buds, leaves, and seedlings, but shows very weak activity in embryos.

Among the remaining enzyme systems, alterations in the expression of isoenzymes occur as follows: the zone $L a p-B$ (see Figure 1) is very weakly expressed in buds and leaves; $S k d h-B$ is weakly expressed also in embryos (not included in
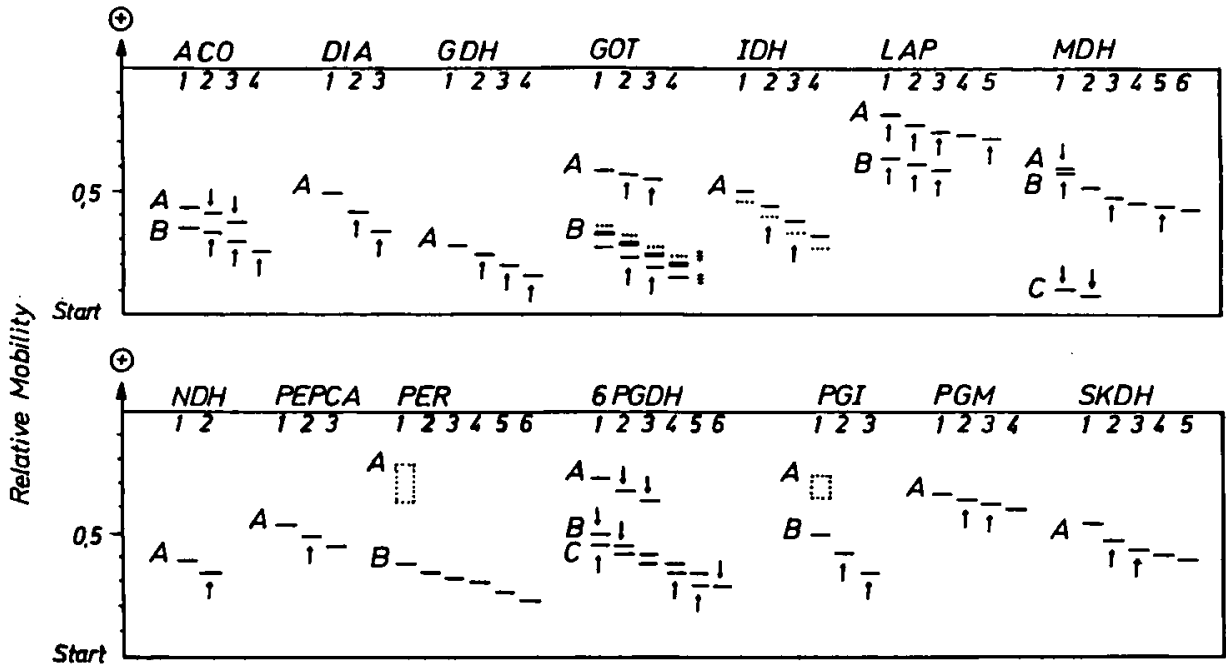

Figure 1. Isoenzyme polymorphism among full-sib families (embryos) and population samples (buds, young leaves) of Fagus sylvatica. Isoenzymes in full-sibs are marked by arrows. LAP-B is very weakly expressed in buds and leaves, PER-B in embryos. For 6PGDH see Figure 2. The scale indicates relative mobility of bands after a 4h run under the given electrophoretic conditions. In population studies (see Materials and Methods), the most frequent types were the following:

$\begin{array}{llll}\text { ACO: } A_{2}, B_{3} & \text { IDH: } A_{3} & \text { PEPCA: } A_{2} & \text { PGM: } A_{3} \\ \text { DIA: } A_{2} & \text { LAP: } A_{2}, B_{2} & \text { PER: } B_{4} & \text { SKDH: } A_{2} \\ \text { GDH: } A_{3} & \text { MDH: } B_{5}, C_{2} & \text { 6PGDH: } A_{2}, B_{2}, C_{4}\left(C_{1}, B_{5}\right) & \\ \text { GOT: } A_{3}, B_{3} & \text { NDH: } A_{2} & \text { PGl: } B_{2} & \end{array}$

* = artifacts in embryos.
Figure 1). In the case of GOT, artifacts appear in embryos within Got-B (see Figure 1) and weakly stained bands between this zone and the start. These bands might refer to a third zone, which is active only in embryos. The available material did not allow inferences on the inheritance of isoenzymes within this Got zone. The other enzyme systems do not show tissue-specific expression of isoenzymes as far as embryos, buds, leaves, and pollen tissues are concerned.

\section{Mode of Inheritance of Isoenzymes}

The available full-sib families did not reveal segregation with respect to PEPCA and NDH. Contrary to embryo tissue, Per-B isoenzymes are clearly expressed in bud and leaf tissue of parental trees and correspond to the ones described by Thiébaut et al. (1982), although three more single bands were found in beech populations in Germany (Müller-Starck 1989 and unpublished data).

In Figure 1, the isoenzyme polymor-

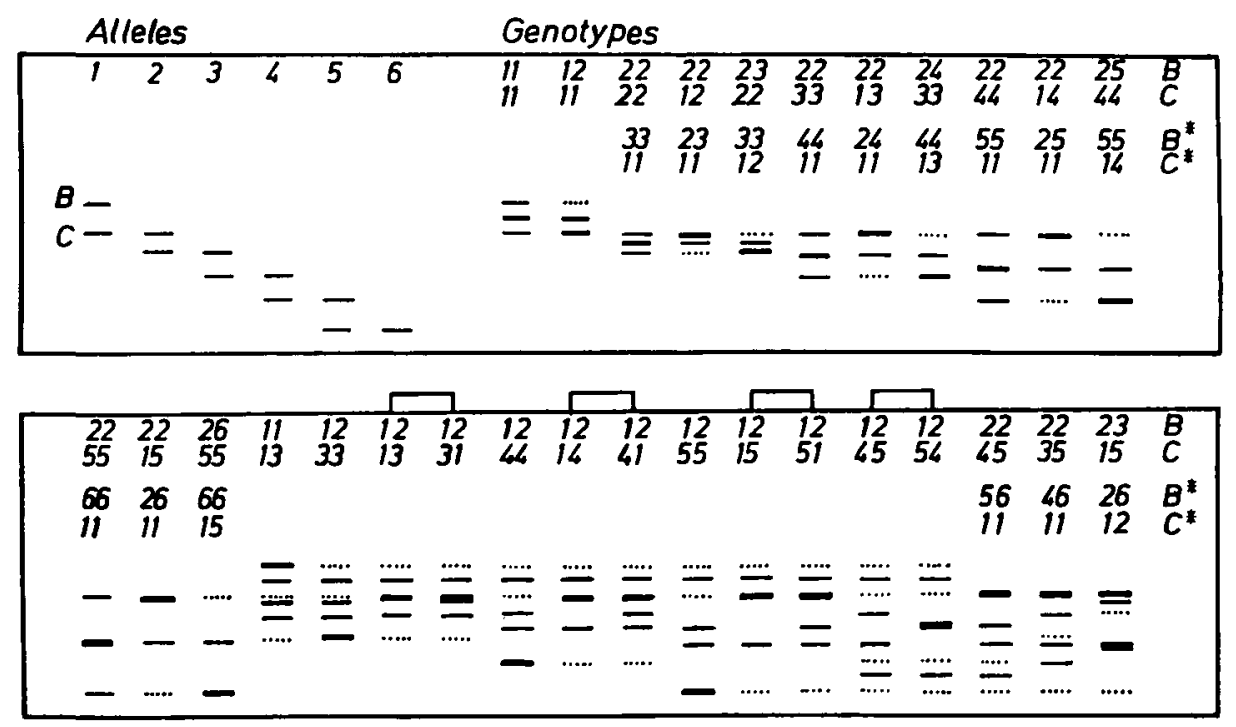

Figure 2. Survey of isoenzyme patterns at $6 P g d h-B$ and $6 P g d h-C$ in Fagus sylvatica and suggested genetic interpretation. Asterisks indicate alternative interpretation as a consequence of $B$ and $C$ bands in identical position. Brackets indicate two-locus types, where different associations of the respective genes in gametes result in different band patterns. 
Table 3. Phenotypes of parents and segregation among full-sib families at the embryo stage

\begin{tabular}{|c|c|c|c|c|c|c|c|c|c|}
\hline \multirow{3}{*}{$\begin{array}{l}\text { Enzyme } \\
\text { system }\end{array}$} & \multicolumn{3}{|c|}{ Parental type } & \multirow{2}{*}{\multicolumn{4}{|c|}{$\begin{array}{l}\text { Segregation within full-sib families: } \\
\text { types and frequency }\end{array}$}} & & \multirow{3}{*}{$\frac{P}{.12}$} \\
\hline & \multicolumn{2}{|l|}{ Female } & \multirow{2}{*}{$\begin{array}{l}\text { Male } \\
\mathrm{A}_{2} \mathrm{~A}_{2} \\
3-80\end{array}$} & & & & & & \\
\hline & \multirow{3}{*}{$\begin{array}{l}A_{2} A_{3} \\
\text { StA-J } \\
B_{2} B_{3} \\
1-80 \\
B_{2} B_{3} \\
\text { SW } 24\end{array}$} & $x$ & & \multirow{3}{*}{$\begin{array}{l}\mathrm{A}_{2} \mathrm{~A}_{2} \\
22 \\
\mathrm{~B}_{2} \mathrm{~B}_{2} \\
6 \\
\mathrm{~B}_{2} \mathrm{~B}_{3} \\
10\end{array}$} & \multirow{3}{*}{$\begin{array}{l}A_{2} A_{3} \\
12 \\
B_{2} B_{3} \\
15 \\
B_{2} B_{4} \\
3\end{array}$} & \multirow{3}{*}{ or } & \multirow{3}{*}{$\begin{array}{l}\mathrm{B}_{3} \mathrm{~B}_{2} \\
\mathrm{~B}_{3} \mathrm{~B}_{3} \\
6\end{array}$} & \multirow{3}{*}{$\begin{array}{l}B_{3} B_{3} \\
6 \\
B_{3} B_{4} \\
11\end{array}$} & \\
\hline & & $x$ & $\begin{array}{l}\mathrm{B}_{2} \mathrm{~B}_{3} \\
2-80\end{array}$ & & & & & & .91 \\
\hline & & $x$ & $\begin{array}{l}\mathrm{B}_{3} \mathrm{~B}_{4} \\
\mathrm{SW} 22\end{array}$ & & & & & & .12 \\
\hline \multirow[t]{2}{*}{ DIA } & $\begin{array}{l}\mathrm{A}_{2} \mathrm{~A}_{3} \\
\text { Lev } 1\end{array}$ & $x$ & $\begin{array}{l}A_{2} A_{2} \\
\text { Lev } 5\end{array}$ & $\begin{array}{l}\mathrm{A}_{2} \mathrm{~A}_{2} \\
14\end{array}$ & $\begin{array}{l}\mathrm{A}_{2} \mathrm{~A}_{3} \\
16\end{array}$ & & & & .86 \\
\hline & $\begin{array}{l}A_{2} A_{3} \\
\text { Lev } 1\end{array}$ & $x$ & $\begin{array}{l}A_{2} A_{2} \\
S W 7\end{array}$ & $\begin{array}{l}\mathrm{A}_{2} \mathrm{~A}_{2} \\
12\end{array}$ & $\begin{array}{l}\mathrm{A}_{2} \mathrm{~A}_{3} \\
18\end{array}$ & & & & .36 \\
\hline \multirow[t]{2}{*}{$\mathrm{GDH}$} & $\begin{array}{l}A_{2} A_{3} \\
6-80\end{array}$ & $x$ & $\begin{array}{l}\mathrm{A}_{3} \mathrm{~A}_{3} \\
5-80\end{array}$ & $\begin{array}{l}\mathrm{A}_{3} \mathrm{~A}_{3} \\
13\end{array}$ & $\begin{array}{l}\mathrm{A}_{2} \mathrm{~A}_{3} \\
7\end{array}$ & & & & .26 \\
\hline & $\begin{array}{l}A_{3} A_{3} \\
10-80\end{array}$ & $x$ & $\begin{array}{l}\mathrm{A}_{3} \mathrm{~A}_{4} \\
\mathrm{Waa}\end{array}$ & $\begin{array}{l}\mathrm{A}_{3} \mathrm{~A}_{3} \\
13\end{array}$ & $\begin{array}{l}\mathrm{A}_{3} \mathrm{~A}_{4} \\
7\end{array}$ & & & & .26 \\
\hline \multirow[t]{4}{*}{ GOT } & $\begin{array}{l}\mathrm{A}_{2} \mathrm{~A}_{3} \\
10-80\end{array}$ & $x$ & $\begin{array}{l}A_{2} A_{3} \\
10-80\end{array}$ & $\begin{array}{l}A_{2} A_{2} \\
6\end{array}$ & $\begin{array}{l}A_{2} A_{3} \\
7\end{array}$ & or & $A_{3} A_{2}$ & $\begin{array}{l}A_{3} A_{3} \\
7\end{array}$ & .39 \\
\hline & $\begin{array}{l}\mathrm{A}_{2} \mathrm{~A}_{3} \\
10-80\end{array}$ & $x$ & $\begin{array}{l}A_{2} A_{3} \\
\text { Lev } 2\end{array}$ & $\begin{array}{l}\mathrm{A}_{2} \mathrm{~A}_{2} \\
7\end{array}$ & $\begin{array}{l}A_{2} A_{3} \\
6\end{array}$ & or & $A_{3} A_{2}$ & $\begin{array}{l}A_{3} A_{3} \\
7\end{array}$ & .18 \\
\hline & $\begin{array}{l}\mathrm{B}_{2} \mathrm{~B}_{3} \\
1-80\end{array}$ & $x$ & $\begin{array}{l}\mathrm{B}_{2} \mathrm{~B}_{2} \\
\mathrm{SW} 8\end{array}$ & $\begin{array}{l}B_{2} B_{2} \\
14\end{array}$ & $\begin{array}{l}B_{2} B_{3} \\
6\end{array}$ & & & & .12 \\
\hline & $\begin{array}{l}B_{2} B_{3} \\
\text { StA-J }\end{array}$ & $x$ & $\begin{array}{l}B_{2} B_{3} \\
\text { SW } 10\end{array}$ & $\begin{array}{l}B_{2} B_{2} \\
6\end{array}$ & $\begin{array}{l}\mathrm{B}_{2} \mathrm{~B}_{3} \\
17\end{array}$ & or & $\mathrm{B}_{3} \mathrm{~B}_{2}$ & $\begin{array}{l}\mathrm{B}_{3} \mathrm{~B}_{3} \\
6\end{array}$ & .69 \\
\hline \multirow[t]{2}{*}{ IDH } & $\begin{array}{l}A_{2} A_{3} \\
\text { StA-H }\end{array}$ & $x$ & $\begin{array}{l}A_{3} A_{3} \\
\text { SW } 19\end{array}$ & $\begin{array}{l}\mathrm{A}_{2} \mathrm{~A}_{2} \\
8\end{array}$ & $\begin{array}{l}A_{2} A_{3} \\
18\end{array}$ & & & & .08 \\
\hline & $\begin{array}{l}A_{2} A_{3} \\
\text { StA-H }\end{array}$ & $x$ & $\begin{array}{l}\mathbf{A}_{2} \mathbf{A}_{3} \\
\text { Lev } 2\end{array}$ & $\begin{array}{l}A_{2} A_{2} \\
4\end{array}$ & $\begin{array}{l}A_{2} A_{3} \\
15\end{array}$ & or & $A_{3} A_{2}$ & $\begin{array}{l}A_{3} A_{3} \\
4\end{array}$ & .44 \\
\hline \multirow[t]{6}{*}{ LAP } & $\begin{array}{l}A_{1} A_{5} \\
1-80\end{array}$ & $x$ & $\begin{array}{l}A_{2} A_{5} \\
\text { SW 5 }\end{array}$ & $\begin{array}{l}A_{1} A_{2} \\
6\end{array}$ & $\begin{array}{l}A_{1} A_{5} \\
5\end{array}$ & & $\begin{array}{l}A_{2} A_{5} \\
6\end{array}$ & $\begin{array}{l}\mathbf{A}_{5} \mathbf{A}_{5} \\
3\end{array}$ & .76 \\
\hline & $\begin{array}{l}A_{1} A_{5} \\
1-80\end{array}$ & $x$ & $\begin{array}{l}A_{2} A_{3} \\
\text { SW } 6\end{array}$ & $\begin{array}{l}A_{1} A_{2} \\
3\end{array}$ & $\begin{array}{l}A_{1} A_{3} \\
8\end{array}$ & & $\begin{array}{l}A_{2} A_{5} \\
3\end{array}$ & $\begin{array}{l}A_{3} A_{5} \\
6\end{array}$ & .35 \\
\hline & $\begin{array}{l}A_{2} A_{5} \\
\text { StA-H }\end{array}$ & $x$ & $\begin{array}{l}\mathrm{A}_{2} \mathrm{~A}_{3} \\
\mathrm{SW} 19\end{array}$ & $\begin{array}{l}\mathrm{A}_{2} \mathrm{~A}_{2} \\
3\end{array}$ & $\begin{array}{l}\mathrm{A}_{2} \mathrm{~A}_{3} \\
8\end{array}$ & & $\begin{array}{l}A_{2} A_{5} \\
6\end{array}$ & $\begin{array}{l}\mathrm{A}_{3} \mathrm{~A}_{5} \\
7\end{array}$ & .51 \\
\hline & $\begin{array}{l}B_{2} B_{3} \\
10-80\end{array}$ & $x$ & $\begin{array}{l}B_{2} B_{3} \\
10-80\end{array}$ & $\begin{array}{l}\mathrm{B}_{2} \mathrm{~B}_{2} \\
6\end{array}$ & $\begin{array}{l}\mathrm{B}_{2} \mathrm{~B}_{3} \\
8\end{array}$ & or & $\mathrm{B}_{3} \mathrm{~B}_{2}$ & $\begin{array}{l}\mathrm{B}_{3} \mathrm{~B}_{3} \\
6\end{array}$ & .70 \\
\hline & $\begin{array}{l}\mathrm{B}_{2} \mathrm{~B}_{3} \\
10-80\end{array}$ & $x$ & $\begin{array}{l}\mathrm{B}_{2} \mathrm{~B}_{2} \\
\text { SW } 8\end{array}$ & $\begin{array}{l}B_{2} B_{2} \\
13\end{array}$ & $\begin{array}{l}\mathrm{B}_{2} \mathrm{~B}_{3} \\
7\end{array}$ & & & & .26 \\
\hline & $\begin{array}{l}\mathrm{B}_{1} \mathrm{~B}_{2} \\
6-80\end{array}$ & $x$ & $\begin{array}{l}\mathrm{B}_{2} \mathrm{~B}_{2} \\
\mathrm{SW} 7\end{array}$ & $\begin{array}{l}\mathrm{B}_{2} \mathrm{~B}_{2} \\
12\end{array}$ & $\begin{array}{l}\mathrm{B}_{1} \mathrm{~B}_{2} \\
8\end{array}$ & & & & .50 \\
\hline \multirow[t]{6}{*}{ MDH } & $\begin{array}{l}\mathrm{B}_{5} \mathrm{~B}_{5} \\
10-80\end{array}$ & $x$ & $\begin{array}{l}B_{1} B_{5} \\
\text { Lev } 2\end{array}$ & $\begin{array}{l}\mathrm{B}_{\mathrm{s}} \mathrm{B}_{\mathrm{s}} \\
21\end{array}$ & $\begin{array}{l}B_{1} B_{5} \\
19\end{array}$ & & & & .88 \\
\hline & $\begin{array}{l}\mathrm{B}_{1} \mathrm{~B}_{5} \\
\text { StA-H }\end{array}$ & $x$ & $\begin{array}{l}\mathrm{B}_{1} \mathrm{~B}_{5} \\
\text { Herz }\end{array}$ & $\begin{array}{l}B_{1} B_{1} \\
4\end{array}$ & $\begin{array}{l}\mathrm{B}_{1} \mathrm{~B}_{5} \\
9\end{array}$ & or & $\mathrm{B}_{5} \mathrm{~B}_{1}$ & $\begin{array}{l}\mathrm{B}_{5} \mathrm{~B}_{5} \\
7\end{array}$ & .57 \\
\hline & $\begin{array}{l}B_{1} B_{5} \\
\text { SW 24 }\end{array}$ & $x$ & $\begin{array}{l}B_{5} B_{5} \\
\text { SW } 14\end{array}$ & $\begin{array}{l}B_{5} B_{5} \\
13\end{array}$ & $\begin{array}{l}\mathrm{B}_{1} \mathrm{~B}_{5} \\
7\end{array}$ & & & & .26 \\
\hline & $\begin{array}{l}C_{1} C_{1} \\
1-80\end{array}$ & $x$ & $\begin{array}{l}\mathrm{C}_{2} \mathrm{C}_{2} \\
\text { SW } 8\end{array}$ & $\begin{array}{l}\mathrm{C}_{1} \mathrm{C}_{2} \\
20\end{array}$ & & & & & 1.00 \\
\hline & $\begin{array}{l}C_{1} C_{2} \\
10-80\end{array}$ & $x$ & $\begin{array}{l}\mathrm{C}_{1} \mathrm{C}_{2} \\
\text { Waa }\end{array}$ & $\begin{array}{l}C_{1} C_{1} \\
5\end{array}$ & $\begin{array}{l}\mathrm{C}_{1} \mathrm{C}_{2} \\
10\end{array}$ & or & $\mathrm{C}_{2} \mathrm{C}_{1}$ & $\begin{array}{l}\mathrm{C}_{2} \mathrm{C}_{2} \\
5\end{array}$ & 1.00 \\
\hline & $\begin{array}{l}\mathrm{C}_{2} \mathrm{C}_{2} \\
\mathrm{SW} 24\end{array}$ & $x$ & $\begin{array}{l}\mathrm{C}_{1} \mathrm{C}_{2} \\
\text { Hum }\end{array}$ & $\begin{array}{l}\mathrm{C}_{2} \mathrm{C}_{2} \\
13\end{array}$ & $\begin{array}{l}\mathrm{C}_{1} \mathrm{C}_{2} \\
7\end{array}$ & & & & .26 \\
\hline \multirow[t]{9}{*}{ 6PGDH } & $\begin{array}{l}A_{2} A_{3} \\
6-80\end{array}$ & $x$ & $\begin{array}{l}\mathrm{A}_{2} \mathrm{~A}_{2} \\
\mathrm{SW} 7\end{array}$ & $\begin{array}{l}A_{2} A_{2} \\
16\end{array}$ & $\begin{array}{l}\mathrm{A}_{2} \mathrm{~A}_{3} \\
14\end{array}$ & & & & .86 \\
\hline & $\begin{array}{l}A_{2} A_{2} \\
S W 16\end{array}$ & $x$ & $\begin{array}{l}A_{2} A_{3} \\
\text { Wee I }\end{array}$ & $\begin{array}{l}A_{2} A_{2} \\
9\end{array}$ & $\begin{array}{l}A_{2} A_{3} \\
11\end{array}$ & & & & .82 \\
\hline & $\begin{array}{l}\mathrm{B}_{1} \mathrm{~B}_{2} \\
\mathrm{SW} 16\end{array}$ & $x$ & $\begin{array}{l}\mathrm{B}_{2} \mathrm{~B}_{2} \\
\text { Herz }\end{array}$ & $\begin{array}{l}\mathrm{B}_{2} \mathrm{~B}_{2} \\
7\end{array}$ & $\begin{array}{l}\mathrm{B}_{1} \mathrm{~B}_{2} \\
13\end{array}$ & & & & .26 \\
\hline & $\begin{array}{l}\mathrm{B}_{1} \mathrm{~B}_{2} \\
\mathrm{SW} 16\end{array}$ & $x$ & $\begin{array}{l}\mathrm{B}_{2} \mathrm{~B}_{2} \\
\text { SW } 11\end{array}$ & $\begin{array}{l}B_{2} B_{2} \\
11\end{array}$ & $\begin{array}{l}\mathrm{B}_{1} \mathrm{~B}_{2} \\
9\end{array}$ & & & & .82 \\
\hline & $\begin{array}{l}\mathrm{B}_{1} \mathrm{~B}_{2} \\
\text { StA-J }\end{array}$ & $x$ & $\begin{array}{l}\mathrm{B}_{2} \mathrm{~B}_{2} \\
3-80\end{array}$ & $\begin{array}{l}\mathrm{B}_{2} \mathrm{~B}_{2} \\
17\end{array}$ & $\begin{array}{l}\mathrm{B}_{1} \mathrm{~B}_{2} \\
6\end{array}$ & & & & $.04^{*}$ \\
\hline & $\begin{array}{l}\mathrm{B}_{2} \mathrm{~B}_{2} \\
\mathrm{StA}-\mathrm{H}\end{array}$ & $x$ & $\begin{array}{l}\mathrm{B}_{2} \mathrm{~B}_{6} \\
\text { Lev } 2\end{array}$ & $\begin{array}{l}\mathrm{B}_{2} \mathrm{~B}_{2} \\
15\end{array}$ & $\begin{array}{l}\mathrm{B}_{2} \mathrm{~B}_{6} \\
5\end{array}$ & & & & $.04^{*}$ \\
\hline & $\begin{array}{l}\mathrm{C}_{1} \mathrm{C}_{4} \\
10-80\end{array}$ & $x$ & $\begin{array}{l}\mathrm{C}_{1} \mathrm{C}_{5} \\
\text { Lev } 2\end{array}$ & $\begin{array}{l}C_{1} C_{1} \\
8\end{array}$ & $\begin{array}{l}C_{1} C_{4} \\
8\end{array}$ & & $\begin{array}{l}\mathrm{C}_{1} \mathrm{C}_{5} \\
13\end{array}$ & $\begin{array}{l}C_{4} C_{5} \\
11\end{array}$ & .66 \\
\hline & $\begin{array}{l}\mathrm{C}_{1} \mathrm{C}_{5} \\
\text { StA-J }\end{array}$ & $x$ & $\begin{array}{l}\mathrm{C}_{1} \mathrm{C}_{4} \\
\text { SW } 10\end{array}$ & $\begin{array}{l}C_{1} C_{1} \\
9\end{array}$ & $\begin{array}{l}\mathrm{C}_{1} \mathrm{C}_{4} \\
7\end{array}$ & & $\begin{array}{l}C_{1} C_{5} \\
9\end{array}$ & $\begin{array}{l}\mathrm{C}_{4} \mathrm{C}_{5} \\
3\end{array}$ & .29 \\
\hline & $\begin{array}{l}C_{1} C_{4} \\
\text { SW } 24\end{array}$ & $x$ & $\begin{array}{l}C_{1} C_{4} \\
\text { SW } 24\end{array}$ & $\begin{array}{l}C_{3} C_{1} \\
5\end{array}$ & $\begin{array}{l}\mathrm{C}_{1} \mathrm{C}_{4} \\
12\end{array}$ & or & $\mathrm{C}_{4} \mathrm{C}_{1}$ & $\begin{array}{l}\mathrm{C}_{4} \mathrm{C}_{4} \\
2\end{array}$ & .37 \\
\hline PGI & $\begin{array}{l}\mathrm{B}_{2} \mathrm{~B}_{3} \\
\text { StA-H }\end{array}$ & $x$ & $\begin{array}{l}B_{2} B_{2} \\
\text { Lev } 2\end{array}$ & $\begin{array}{l}\mathrm{B}_{2} \mathrm{~B}_{2} \\
10\end{array}$ & $\begin{array}{l}\mathrm{B}_{2} \mathrm{~B}_{3} \\
11\end{array}$ & & & & 1.00 \\
\hline PGM & $\begin{array}{l}\mathrm{A}_{2} \mathrm{~A}_{3} \\
\text { StA-J }\end{array}$ & $x$ & $\begin{array}{l}\mathrm{A}_{2} \mathrm{~A}_{3} \\
\mathrm{SW} 22\end{array}$ & $\begin{array}{l}A_{2} A_{2} \\
5\end{array}$ & $\begin{array}{l}A_{2} A_{3} \\
18\end{array}$ & or & $A_{3} A_{2}$ & $\begin{array}{l}A_{3} A_{3} \\
6\end{array}$ & .49 \\
\hline SKDH & $\begin{array}{l}A_{2} A_{2} \\
\text { SW } 24\end{array}$ & $x$ & $\begin{array}{l}A_{2} A_{3} \\
S W 21\end{array}$ & $\begin{array}{l}A_{2} A_{2} \\
13\end{array}$ & $\begin{array}{l}\mathbf{A}_{2} \mathbf{A}_{3} \\
7\end{array}$ & & & & .26 \\
\hline
\end{tabular}

$* P<.05$.

The supposed gene loci are designated by capital letters, the supposed alleles by numbers (for nomenclature see Figure 1). phism among full-sib families is accomplished by isoenzymes that were monitored in population studies (see legend). The inheritance of the latter is suggested because each of these isoenzymes appears in single bands or combinations of bands, which does not refute the hypothesis of a phenotypic representation of homozygotes (single bands) or heterozygotes (double bands in monomeric systems, additional hybrid band(s) according to the enzyme structure) at single loci. This also holds for all combinations between the identified alleles and the suggested ones. There is no contradiction between the present interpretation and the inheritance of those systems in other tree species (see analogous segregation analyses in deciduous trees such as poplars (Müller-Starck 1992) and oaks (Müller-Starck and Hattemer 1990).

\section{Peculiarities of Enzyme Systems}

Artifacts are expressed in Got-B (embryos) and Idh-A (see Figure 1). In the case of Lap-A, former studies monitored four different isoenzyme bands (e.g., Kim 1980; Müller-Starck 1985; Thiébaut et al. 1982) instead of five. The reason is the very close location of $A 4$ (very rare) and $A 5$, which therefore might have been pooled in earlier investigations. $\mathrm{MDH}-\mathrm{A}$ is monomorphic until now in Fagus sylvatica but polymorphic in other species (e.g., Müller-Starck 1992). The system of PEPCA is faintly stained. PGI-A is excluded from further interpretation because of insufficient substrate specificity. Skdh-B is omitted because faint staining intensity and very closely located isoenzyme variants substantially impede the genetic interpretation. There is an overlap between Skdh-A and Skdh-B. Under the given electrophoretic conditions, Skdh-A is intensively stained so that Skdh-B will not interfere.

\section{Interpretation of the 6PGDH System}

The most complex system is $6 \mathrm{PGDH}$, because 6Pgdh-B and 6Pgdh-C form interlocus hybrids (heterodimers) and show substantial overlap. The present interpretation of the 6PGDH polymorphism is based on both segregation among full-sibs and population data. Figure 2 shows problems related to genotyping at the loci $6 P g d h-B$ and $6 P g d h-C$. These problems are caused by the fact that bands expressed by these loci can be located in the zymograms in identical or nearly identical position. Therefore, in several cases, zy- 
Table 4. Enzyme system, enzyme structure, and enzyme coding gene loci in embryos, buds, young leaves, and pollen of Fagus sylvatica $\mathrm{L}$. (ACP, ${ }^{\circ} \mathrm{EST}^{b}$, and monomorphic zones excluded)

\begin{tabular}{lll} 
Enzyme system (EC no.) & Structure & Gene locus \\
\hline Aconitase (4.2.1.3) & Monomeric & Aco-A, Aco-B \\
Diaphorase (1.6.99) & Tetrameric & Dia-A \\
Glutamate dehydrogenase (1.4.1.3.) & Polymeric & Gdh-A \\
Glutamate oxaloacetate transaminase (2.6.1.1) & Dimeric & Got-A, Got-B \\
Isocitrate dehydrogenase (1.1.1.42) & Dimeric & Idh-A \\
Leucine aminopeptidase (3.4.11.1) & Monomeric & Lap-A, Lap-Bc \\
Malate dehydrogenase (1.1.1.37) & Dimeric & Mdh-B, Mdh-C \\
NADH-dehydrogenase (1.6.99.3) & Polymeric & Ndh-A \\
Peroxidase (1.11.1.7) & Monomeric & Per-Bd, \\
Phosphoenolpynute carboxylase (4.1.1.31) & Dimeric & Pepca-A \\
6-Phosphogluconate dehydrogenase (1.1.1.44) & Dimeric & $6 P g d h-A, 6 P g d h-B$, \\
& & $6 P g d h-C$ \\
Phosphoglucose isomerase (5.3.1.9) & Dimeric & Pgi-B \\
Phosphoglucomutase (5.4.2.2) & Monomeric & Pgm-A \\
Shikimate dehydrogenase (1.1.1.25) & Monomeric & Skdh-A
\end{tabular}

' Acid phosphatase (3.1.3.2)

${ }^{\circ}$ Esterase (3.1.1.1).

c Only in embryos.

${ }^{d}$ Not in embryos.

' Inferred from population studies (no segregation among full-sibs).

$f=P x_{2}$ (Thiébaut et al. 1982). mograms can be interpreted in two different ways. The respective alternative for genotyping at these two loci is indicated in Figure 2 by asterisks.

The given genetic interpretation of complex band patterns is strongly suggested because of clearly visible differences in staining intensities. This is evident particularly with respect to the three-banded isoenzymes (see upper right and lower left part of Figure 2). The different intensities are a consequence of the overlap of single bands and appear clearly in the zymograms. The designation of the remaining band patterns follows these interpretations. Visualization of zymograms was tested separately by employing gels with 20 $\mathrm{cm}$ migration distance. The segregations in Table 3 and five other segregations within 6Pgdh-B and 6Pgdh-C (not included in Table 3 due to lack of space) did not reveal significant deviations from the expected Mendelian proportions.

In Figure 2 band patterns monitored in each of the studied beech populations are surveyed. Many other two-locus combinations can be expected but seem to be extremely rare in beech populations. We never found two-locus types that comprised more than eight single bands. It appears that the expression of the diploid two-locus genotype is not independent from the association of the respective genes in gametes. In Figure 2 such cases are indicated by brackets. Linkage can be expected to account for this phenomenon
Table 5. Survey of tested two-locus combinations and number of available full-sib familles (deviations are statistically not significant)

\begin{tabular}{|c|c|c|c|c|c|c|c|c|c|c|c|c|c|c|c|c|c|}
\hline \multirow{2}{*}{$\begin{array}{l}\text { Gene } \\
\text { locus }\end{array}$} & \multicolumn{2}{|c|}{ Aco } & \multirow{2}{*}{$\begin{array}{l}\text { Dia } \\
A\end{array}$} & \multirow{2}{*}{$\begin{array}{l}G d h \\
A\end{array}$} & \multicolumn{2}{|c|}{ Got } & \multirow{2}{*}{$\begin{array}{l}/ d h \\
A\end{array}$} & \multicolumn{2}{|c|}{ Lap } & \multicolumn{2}{|c|}{$M d h$} & \multicolumn{3}{|c|}{$6 P g d h$} & \multirow{2}{*}{$\begin{array}{l}P_{\mathbf{g} i} \\
B\end{array}$} & \multirow{2}{*}{$\begin{array}{l}P g m \\
A\end{array}$} & \multirow{2}{*}{$\begin{array}{l}S k d h \\
A\end{array}$} \\
\hline & $A$ & $B$ & & & $A$ & $B$ & & $A$ & $B$ & $B$ & $C$ & $A$ & $B$ & $C$ & & & \\
\hline$A C O-A$ & & & & & 1 & 1 & & & & & & & 1 & & & & \\
\hline$A C O-B$ & & & & & & 2 & & 1 & & 1 & 1 & & & 1 & & & 1 \\
\hline$D i a-A$ & & & & & & & & 1 & 1 & & 1 & 2 & & & & & \\
\hline$G d h-A$ & & & & & 1 & 1 & & & & & 1 & $\overline{1}$ & 1 & 1 & & & \\
\hline Got-A & & & & & & 9 & 2 & 2 & 1 & 3 & 6 & 1 & 2 & 3 & & & 1 \\
\hline Got-B & & & & & & & 3 & 11 & 1 & 9 & 9 & 1 & 2 & 5 & & 1 & 3 \\
\hline$I d h-A$ & & & & & & & & 3 & 1 & 3 & 2 & 1 & 1 & 2 & 1 & & 3 \\
\hline$L a p-A$ & & & & & & & & & 1 & 8 & 5 & 1 & 1 & 1 & & & 1 \\
\hline$L a p-B$ & & & & & & & & & & & 1 & 2 & & & & & \\
\hline$M d h-B$ & & & & & & & & & & & 6 & 2 & 2 & 5 & & & 2 \\
\hline$M d h-C$ & & & & & & & & & & & & 2 & 1 & 4 & & & 3 \\
\hline $6 P g d h-A$ & & & & & & & & & & & & & 1 & 3 & 1 & & 1 \\
\hline $6 P g d h-B$ & & & & & & & & & & & & & & 1 & 1 & & 1 \\
\hline 6Pgdh-C & & & & & & & & & & & & & & & $i$ & & 3 \\
\hline Pgi-B & & & & & & & & & & & & & & & & & \\
\hline$P g m-A$ & & & & & & & & & & & & & & & & & \\
\hline$S \boldsymbol{k} d h \cdot A$ & & & & & & & & & & & & & & & & & \\
\hline
\end{tabular}

but could not be tested explicitly because of the lack of heterozygous two-locus combinations among the parental trees.

\section{Segregation among Full-sib Families}

In Table 3, for each enzyme system except NDH, PEPCA, and PER, a set of segregations is listed of those isoenzyme bands marked by arrows in Figure 1. The given examples include all cases in which deviations from the expected Mendelian segregations were at maximum.

With two exceptions (6PGDH-B: StA-J $\times$ $3-80$, StA-H $\times$ Lev 2 , see Table 3 ), all tested segregations were in statistical agreement with the expected Mendelian ones. If all tests are taken into account (Table 3 surveys 37 of more than 100), one would expect more than two tests to yield values less than .05 just by chance. Besides that, viability selection in early life stages cannot be excluded. Lacking viability of embryos may account for the weak expression of bands of a few embryos so that zymograms could not unequivocally be interpreted. This is why the actual sample sizes of one and the same full-sib family can vary slightly among enzyme systems.

All other segregations do not refute the hypothesis of a one-locus control for those isoenzyme bands that appear within the same zone in the zymograms (for 6PGDH, see the preceding discussion). It can be concluded that in all studied enzyme systems homozygotes are represented in the zymograms by single bands; heterozygotes are represented by combinations of double bands in the case of monomeric enzyme systems, by three-banded types in dimeric systems (one hybrid band), by five-banded types in tetrameric systems (three hybrid bands), and by multibanded types in case of polymeric systems. All alleles appear to be expressed codominantly. Lack of activity (null alleles) was not apparent in full-sibs but cannot be excluded (for LAP, see Kim 1979).

Table 4 show 17 polymorphic loci that are part of segregation studies and three more loci (Ndh-A, Per-A, Pepca-A) in which the variation is inferred from population studies. At present, the total number of genes (alleles) monitored at these 20 loci in full-sib families and population studies is 78 , or 3.9 per locus. This value exceeds the corresponding values of many other tree species (see Müller-Starck et al. 1992).

\section{Recombination Studies}

The available two-locus combinations and the results of a testing of uniformity of the 
Table 6. Segregation among two-locus genotypes and results of a statistical testing of hypotheses 1 and 4

\begin{tabular}{|c|c|c|c|c|c|c|c|c|}
\hline \multirow{2}{*}{$\begin{array}{l}\text { Gene loci } \\
\text { (pair) }\end{array}$} & \multicolumn{2}{|c|}{$\begin{array}{l}\text { Parental } \\
\text { genotypes }\end{array}$} & \multicolumn{3}{|c|}{$\begin{array}{l}\text { 2-locus segregations within } \\
\text { full-sib families }\end{array}$} & \multicolumn{2}{|l|}{$P$} & \multirow{2}{*}{$\begin{array}{l}\text { Proportion } \\
\text { of } \\
\text { supposed } \\
\text { recombi- } \\
\text { nants (\%) }\end{array}$} \\
\hline & Female & Male & Genotypes & Frequency & $N$ & Hyp. 1 & Нур. 4 & \\
\hline $\begin{array}{l}\text { Gdh-A } \\
\text { 6Pgdh-C }\end{array}$ & $\begin{array}{l}6-80 \times \\
12 \\
11\end{array}$ & $\begin{array}{r}5-80 \\
12 \\
15\end{array}$ & $\begin{array}{llll}12 & 22 & 12 & 22 \\
15 & 15 & 11 & 11\end{array}$ & 4:8:3:5 & 20 & 0.512 & 0.824 & 45 \\
\hline $\begin{array}{l}\text { Got-A } \\
\text { Got-B }\end{array}$ & $\begin{array}{l}\text { SW } 16 \times \\
33 \\
33\end{array}$ & $\begin{array}{c}\text { SW } 18 \\
23 \\
23\end{array}$ & $\begin{array}{llll}32 & 33 & 32 & 33 \\
32 & 32 & 33 & 33\end{array}$ & $7: 3: 6: 4$ & 20 & 0.630 & 0.824 & 45 \\
\hline $\begin{array}{l}\text { Got-B } \\
M d h-C\end{array}$ & $\begin{array}{l}\text { SW } 16 \times \\
33 \\
22\end{array}$ & $\begin{array}{r}\text { Wag } \\
23 \\
12\end{array}$ & $\begin{array}{llll}32 & 33 & 32 & 33 \\
21 & 21 & 22 & 22\end{array}$ & $4: 4: 7: 5$ & 20 & 0.838 & 0.824 & 45 \\
\hline $\begin{array}{l}\text { Lap-B } \\
6 P g d h-A\end{array}$ & $\begin{array}{l}\text { Lev } 1 \times \\
12 \\
23\end{array}$ & $\begin{array}{r}\mathrm{SW} 7 \\
11 \\
22\end{array}$ & $\begin{array}{llll}11 & 21 & 11 & 21 \\
22 & 22 & 32 & 32\end{array}$ & 9:3:3:5 & 20 & 0.219 & 0.115 & 30 \\
\hline $\begin{array}{l}\text { Mdh-B } \\
6 P g d h-C\end{array}$ & \multicolumn{2}{|c|}{$\begin{array}{lr}10-80 \times \text { Lev } 2 \\
55 & 15 \\
14 & 15\end{array}$} & $\begin{array}{llll}51 & 51 & 51 & 51 \\
11 & 15 & 41 & 45 \\
55 & 55 & 55 & 55 \\
11 & 15 & 41 & 45\end{array}$ & $\begin{array}{l}2: 6: 4: 9 \\
6: 8: 4: 1\end{array}$ & 40 & 0.224 & 0.636 & 45 \\
\hline $\begin{array}{l}\text { Mdh-C } \\
\text { Got-B }\end{array}$ & $\begin{array}{l}\text { SW } 24 \times \\
12 \\
23\end{array}$ & $\begin{array}{c}\text { Wag } \\
22 \\
33\end{array}$ & $\begin{array}{llll}12 & 22 & 12 & 22 \\
23 & 23 & 33 & 33\end{array}$ & $4: 7: 3: 6$ & 20 & 0.630 & 1.000 & 50 \\
\hline $\begin{array}{l}\text { 6Pgdh-A } \\
\text { 6Pgdh-C }\end{array}$ & $\begin{array}{l}\text { StA-H X } \\
22 \\
11\end{array}$ & $\begin{array}{c}\text { Lev } 2 \\
2.3 \\
15\end{array}$ & $\begin{array}{llll}22 & 23 & 22 & 23 \\
11 & 11 & 15 & 15\end{array}$ & $6: 9: 3: 2$ & 20 & 0.136 & 0.503 & 40 \\
\hline $\begin{array}{l}\text { 6Pgdh-B } \\
M d h-B\end{array}$ & $\begin{array}{l}\mathrm{SW} 16 \times \\
12 \\
15\end{array}$ & $\begin{array}{c}\text { Wag } \\
22 \\
55\end{array}$ & $\begin{array}{llll}12 & 22 & 12 & 22 \\
15 & 15 & 55 & 55\end{array}$ & $7: 5: 6: 2$ & 20 & 0.424 & 0.824 & 45 \\
\hline $\begin{array}{l}\text { Pgi-B } \\
6 P g d h-B\end{array}$ & $\begin{array}{l}\text { StA-H X } \\
23 \\
22\end{array}$ & $\begin{array}{l}\text { Lev } 2 \\
22 \\
26\end{array}$ & $\begin{array}{llll}22 & 32 & 22 & 32 \\
22 & 22 & 26 & 26\end{array}$ & $6: 9: 3: 2$ & 20 & 0.136 & 0.503 & 40 \\
\hline $\begin{array}{l}\text { Pgi-B } \\
\text { Skdh-A }\end{array}$ & $\begin{array}{l}\text { StA-H } \times \\
23 \\
23\end{array}$ & $\begin{array}{l}\text { Lev } 2 \\
22 \\
22\end{array}$ & $\begin{array}{llll}22 & 32 & 22 & 32 \\
22 & 22 & 32 & 32\end{array}$ & $3: 5: 6: 7$ & 21 & 0.701 & 1.000 & 48 \\
\hline
\end{tabular}

Alleles are printed in italics, observed frequencies in boldface.

two-locus genotypes are given in Table 5 . A set of examples is given in Table 6.

Hypotheses 1 and 4 refer to the frequency distribution of all two-locus types and of the recombinant and the nonrecombinant types (see Materials and Methods). For tests on hypotheses 2 and 3 see Table 3.

Because there was no two-locus segregation to reject hypothesis 4 , recombination values could not be calculated. Therefore, Table 6 shows the proportion of supposed recombinants. In most cases this value is close to $50 \%$. Only 75 of the possible 136 two-locus combinations (17 gene loci) could be analyzed, however, due to the homozygous condition of the parental trees. The main problem is the lack of haploid material so that, in many cases, reciprocal types cannot be distinguished and must be pooled. Moreover, small sample sizes severely limit the information pool of the present material.
Linkage is not indicated in the present material. A study on recombination with respect to Acp-A and Lap-A in beech (Kim 1979,1980 ) results in the same conclusion. As in beech, linkage could not be verified in full-sib families of Quercus petraea and Q. robur (Müller-Starck and Hattemer are also the subject here. To our knowledge, there are no other studies on recombination in the genera Fagus, Castanea, or Quercus ( $n=12$ chromosomes). By employing Ap-A,B,C (Ap = aminopeptidase) and Got- $B$, Linares Bensimon (1984) observed linkage between Ap-A and Ap-B in Alnus glutinosa ( $n=14$ chromosomes). In populations of Alnus crispa, Bousquet et al. (1987) studied the frequency of gametes in the pollen pool with respect to eight polymorphic loci (excluding Lap-A,B). A significant nonrandom association of alleles at two-locus combinations could not be verified. 1990); this study referred to 15 loci that
As in many other tree species, in beech further investigations are required to study recombination in more detail. At present, there is no evidence for a clustered location of the analyzed gene loci at single chromosomes.

\section{References}

Bergmann, F and Scholz F, 1989. Selection effects of air pollution in Norway spruce (Picea abies) populations. In: Genetic aspects of air pollutants in forest tree populations (Scholz F, Gregorius HR, and Rudin D, eds). Berlin: Springer-Verlag; 143-160.

Bousquet JJ, Cheliak WM, and Lalonde M, 1987. Allozyme variation in natural populations of green alder [Alnus crispa (Ait.) Pursh.] in Quebec. Genome 29:345352.

Kim ZS, 1979. Inheritance of leucine aminopeptidase and acid phosphatase isoenzymes in beech (Fagus syl vatica $\mathrm{L}$.). Silvae Genet 28:68-71.

Kim ZS, 1980. Veränderungen der genetischen Struktur von Buchenpopulationen durch Viabilitätsselektion im Keimlingsstadium. Göttingen Res Notes Forest Genet 3:1-88.

Linares Bensimon C, 1984. Versuche zur Viabilitätsselektion an Enzym-Genloci bei Alnus glutinosa (L.) Gaertn. Göttingen Res Notes Forest Genet 7:1-13.

Lundkvist K, 1979. Allozyme frequency distribution in four Swedish populations of Norway spruce (Picea abies K.). Hereditas 90:127-143

Merzeau D, Di Giusto F, Comps B, Thiébaut B, Letouzey $J$, and Cuguen J, 1989. Genetic control of isoenzyme systems and heterogeneity of pollen contribution in beech (Fagus sylvatica L.). Silvae Genet 38:195-201.

Müller-Starck G, 1985. Genetic differences between "tolerant" and "sensitive" beeches (Fagus sylvatica L.) in an environmentally stressed adult forest stand. Silvae Genet 34:241-247.

Müller-Starck G, 1989. Genetic implications of environmental stress in adult forest stands of Fagus sylvatica $\mathrm{L}$. In: Genetic aspects of air pollutants in forest tree populations (Scholz F, Gregorius H-R, and Rudin D, eds). Berlin: Springer-Verlag; 127-142.

Müller-Starck G, 1992. Genetic control of isoenzymes in poplars of the Tacamahaca section and hybrids. Silvae Genet 41:87-95.

Müller-Starck G, Baradat Ph, and Bergmann F, 1992. Genetic variation in European tree species. New For ests 6:23-47.

Müller-Starck G, and Hattemer HH, 1990. Genetics and breeding of oaks. Final Report, MA IB-0012-D. Brussels: Commission European Communities, DG XII.

Müller-Starck G and Ziehe M, 1991. Genetic variation in populations of Fagus sylvatica L., Quercus robur L., and Quercus petraea Liebl. in Germany. In: Genetic variation in European populations of forest trees (Müller-Starck $\mathbf{G}$, and Ziehe $M$, eds). Frankfurt: Sauerländer's Verlag; 125-140.

Nielsen $P$ and Schaffalitzky de Muckadell M, 1954 Flower observation and controlled pollinations in $\mathrm{Fa}$ gus. Silvae Genet 3:6-17.

Poulik M, 1957. Starch gel electrophoresis in a dis continuous system of buffers. Nature 180:1477-1478.

Thiébaut B, Lumaret $\mathrm{R}$, and Vernet $\mathrm{Ph}, 1982$. The bud enzymes of beech (Fagus sylvatica L.): genetic distinction and analysis of polymorphism in several French populations. Silvae Genet 31:51-60. 